\title{
ROLE OF RIGID BRONCHOSCOPY IN MASSIVE HAEMOPTYSIS
}

\author{
Ng TH ${ }^{\#}$, How SH ${ }^{\#}$ Kuan YC ${ }^{\#}$, R Ahmad MS*, Fauzi AR ${ }^{\#}$ \\ \#Department of Internal Medicine, Kulliyyah of Medicine, International Islamic University Malaysia, Kuantan, Malaysia. \\ ${ }^{*}$ Department of Otorhinolaryngology, Kulliyyah of Medicine, International Islamic University Malaysia, Kuantan, Malaysia.
}

\begin{abstract}
:
Massive haemoptysis can occur in lung abscess. Massive haemoptysis itself may be life threatening due to asphyxiation or respiratory failure secondary to acute large airway obstruction by blood clots. Prompt removal of the obstructing blood clots save life. We describe a case of lung abscess causing massive haemoptysis resulting in acute airway obstruction which required rigid bronchoscopy to remove the huge blood clot. (JUMMEC2010; 13(2): 107-110)
\end{abstract}

KEYWORDS: Massive hemoptysis, airway obstruction, rigid bronchoscopy

\section{Introduction}

Massive haemoptysis can be caused by numerous pathologies of the lower respiratory tract. The main causes include bronchiectasis, tuberculosis, lung cancer, chronic inflammatory diseases and cystic fibrosis. Massive haemoptysis can result in acute endobronchial obstruction leading to acute respiratory failure and even mortality in $50-100 \%$ of the patients affected (1). The blood clots which obstruct the major airway can be removed by various methods. We describe a case of lung abscess causing massive haemoptysis resulting in acute respiratory failure which required rigid bronchoscopy for blood clot evacuation.

\section{Case Report}

An 83-year-old gentleman, who was a heavy smoker, presented to a district hospital with one week of fever, productive cough and haemoptysis. Chest X-ray (CXR) showed pneumonic changes at the left basal region. He was treated for pneumonia with tablet cefuroxime $500 \mathrm{mg}$ twice daily for 10 days. Three months later, he was readmitted to a similar district hospital with two weeks of fever and productive cough with frank haemoptysis. He had loss of appetite and weight. CXR revealed a cavitating lung lesion suggestive of a left lung abscess at the left lower lobe (Figure 1). Computed tomography of the thorax was done and showed a thick-walled cavitating lesion with air-fluid level in the apical segment of the left lower lobe with a small solitary pretracheal lymph node. His condition improved with i.v. Co-amoxiclav $1.2 \mathrm{~g}$ three times a day and was discharged with a 4-week course of oral Co-amoxiclav $625 \mathrm{mg}$ twice daily. One month later, he presented to us again with fever and massive haemoptysis which was associated with hypotension (blood pressure was $80 / 50 \mathrm{mmHg}$ ). He was resuscitated and stabilized expectantly. He had worsening cough with frank haemoptysis over the night. This was associated with dyspnoea and hypoxemia which required high-flow oxygen supplement to maintain an oxygen saturation of $90-92 \%$.

On physical examination, the patient was thin, pale and was using accessory muscles of respiration. There was no finger clubbing or lymphadenopathy. His respiratory rate was 30 breaths per minute and body temperature was $38^{\circ}$ Celsius. Examination of the

Correspondence:

$\mathrm{Ng}$ Teck Han

Kulliyyah of Medicine, International Islamic University Malaysia,

27510 Kuantan

Pahang, Malaysia.

E-mail:ngteckhan@hotmail.com 


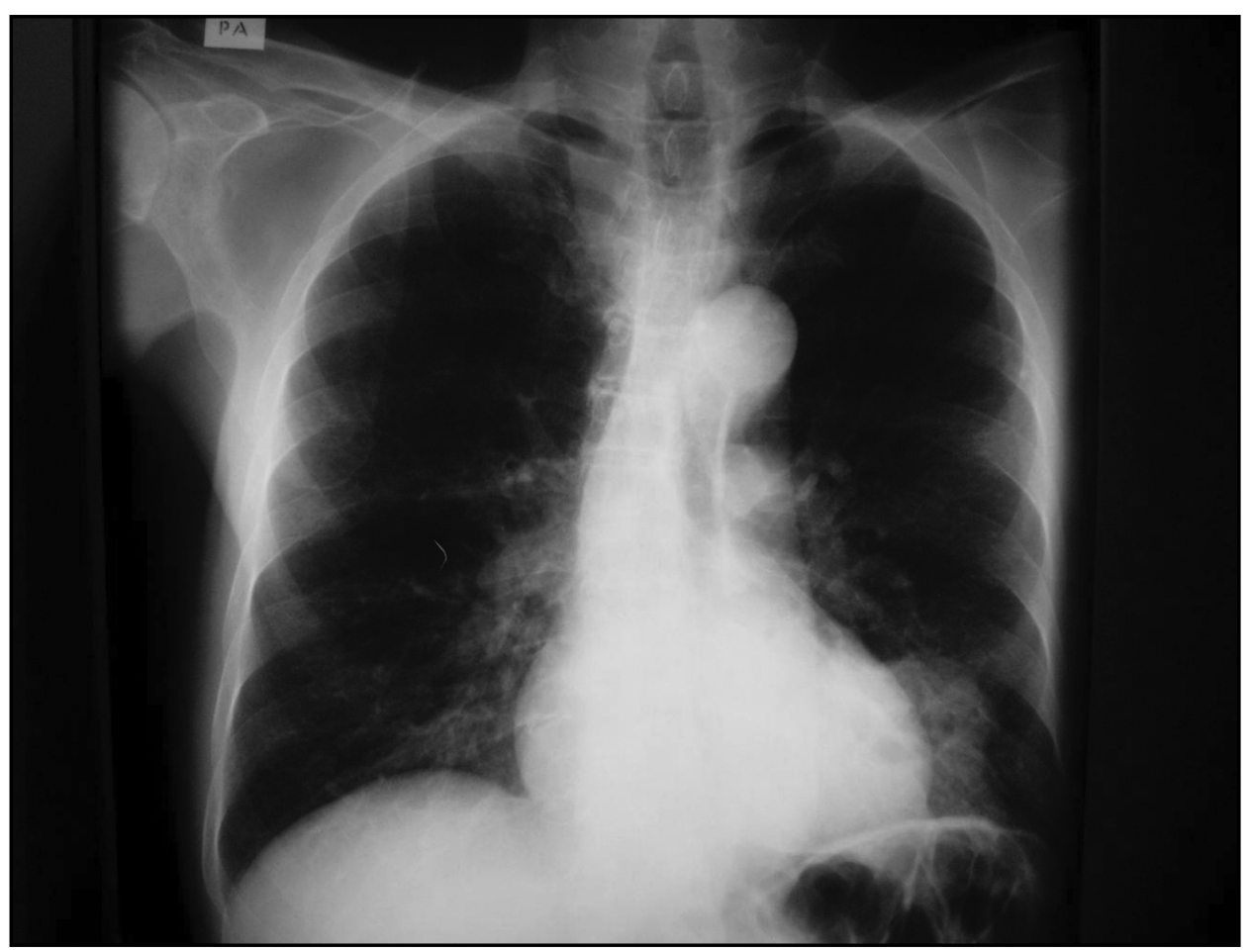

Figure 1: Chest X-ray shows a cavitating lung lesion suggestive of lung abscess in the left lower lobe.

lungs revealed signs of left lung collapse. There was no rhonchi and the right lung was normal. Other systemic examinations were otherwise unremarkable.

His haemoglobin level was $8.8 \mathrm{~g} / \mathrm{dL}$, total white cell count was $27 \times 10^{9} / \mathrm{L}$ with neutrophilia and platelet count was $165 \times 10^{3} / \mathrm{L}$. His coagulation profile, liver function test and renal profile were normal. Repeated CXR showed total left lung collapse. All work-up for tuberculosis (acid fast bacilli smears of sputum, culture for mycobacterium tuberculosis and tuberculin test were negative). His sputum and blood cultures were negative.

He was given intravenous Co-amoxiclav $1.2 \mathrm{~g}$ eight hourly on admission. An urgent flexi-bronchoscopy showed a blood clot obstructing the entire left main bronchus (Figure 2). Endobronchial lavage and forceps extraction were unsuccessful in removing the entire blood clot. In view of persistent lung collapse and suboptimal oxygenation, we proceeded with rigid bronchoscopy, which was performed by an otorhinolaryngologist. It was done in a general operation theater under total intravenous anesthesia with propofol infusion and spontaneous respiration. The patient was hyperventilated before topical anesthesia (lidocaine 2\%) was sprayed into the larynx. The rigid bronchoscope was introduced through the glottic inlet with delicate manipulation, where the bevel end of the scope was positioned vertically. Then the scope was advanced into the mid-trachea. The carina was identified. The pathology mainly involved the left main bronchus, which was totally occluded by the blood clot. The blood clot was removed in piecemeal manner by using the foreign body removing forceps and suctioning. Concomitant chest percussion on the left side was employed to help dislodge any impacted blood clot in the secondary bronchus. The air entry of the left lung improved remarkably and oxygenation normalized after removal of the blood clot. The flexi-bronchoscope was introduced through the rigid bronchoscope and showed no endobronchial lesion, but thick pus discharge was seen at the left lower lobe bronchus. Bronchioalveolar lavage (BAL) was done and culture grew Pseudomonas aeruginosa. BAL cytology and fungal staining were negative. Repeated CXR showed re-expansion of the left lung with persistent lung abscess of the left lower lobe.

His antibiotics was changed to intravenous ciprofloxacin $500 \mathrm{mg}$ twice daily. Unfortunately, the patient continued to have persistent mild to moderate 


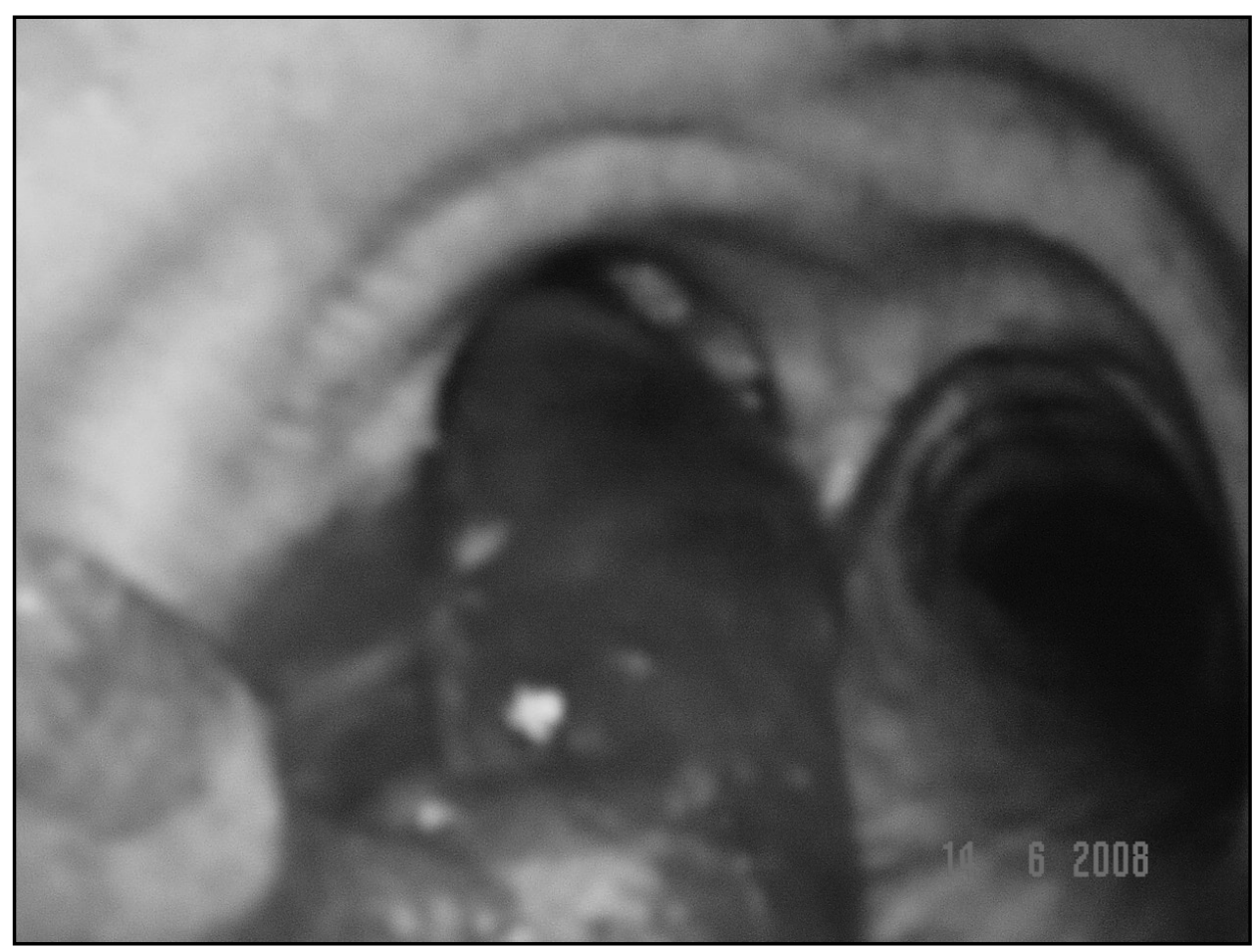

Figure 2: Flexi-bronchoscopic view showing a huge blood clot obstructing left main bronchus.

haemoptysis in the ward. He was then referred to tertiary hospital for arterial embolization which was not available in our hospital in that point of time. Unfortunately, he succumbed to hospital acquired infection with uncontrolled septicemia one week later. He did not have any episodes of massive haemoptysis after the rigid bronchoscopy.

\section{Discussion}

Acute endobronchial obstruction can be caused by blood clots from massive haemoptysis. Massive haemoptysis has been defined as the expectoration of $300 \mathrm{ml}$ or more of blood per day (1). Airway obstruction caused by the presence of blood clots has been noted as a complication of bronchiectasis, tuberculosis, mitral stenosis, pulmonary infarction, pulmonary arteriovenous malformation, sarcoidosis, bronchial carcinoma, and intrathoracic trauma (2).

Massive haemoptysis may be life-threatening due to asphyxiation or respiratory failure secondary to acute large airway obstruction by blood clots. The source of massive haemoptysis is usually located in the bronchial artery (90\%), pulmonary arteries (5\%) and the remaining $5 \%$ in the aorta (aortobronchial fistulae or rupture of aortic aneurysms) or the systemic arterial circulation (branches serving the lungs) (1). Massive haemoptysis is associated with mortality rate of $50 \%$ to $100 \%$ (1). Our patient had massive haemoptysis secondary to lung abscess resulting in acute airway obstruction with respiratory failure. Approximately $30 \%$ of cases with acute airway obstruction by blood clots reported in the literature had no evidence of preceding haemoptysis (2). This is because tracheobronchial mucosal damage from infection or tumor may result in subclinical endobroncial bleeding with subsequent clot formation (2). Therefore, diagnosis of endobronchial clot should always be considered when the patient presents with a sudden onset of acute airway obstruction despite the absence of haemoptysis.

Our patient had chronic constitutional symptoms and haemoptysis which made bronchogenic carcinoma a likely differential diagnosis. The reported incidence of carcinoma in patients who presented with lung abscess ranged from $7.6-17.5 \%$ (3). We had considered surgical option for the patient in view of persistent haemoptysis and risk of concomitant lung cancer. However the patient refused any further intervention. Lung abscess can be associated with post-inflammatory 
vascular malformation (pseudoaneurysm) causing massive haemoptysis (4). One study showed that arterial coil embolization of the feeding arteries successfully stops bleeding in $91 \%$ of the cases (1). This procedure is however not available in our hospital in that point of time.

There are many ways of removing the obstructing endobronchial blood clot. Flexible bronchoscopy should be used as an initial effort in removing the blood clots by means of saline lavage, suctioning and forceps extraction through the working channel, either en bloc or in piecemeal fashion. It is particularly difficult to remove a large clot as in our patient, despite multiple attempts of saline lavage and forceps extraction. This is because the clot is still soft and it breaks easily on grasping. Rigid bronchoscopy should be considered if flexible bronchoscope failed to remove the clot. It allows greater access of the airway and thus offers better suction capability and forceps extraction of the larger blood clot by bigger forceps. It also offers superior airway management should brisk bleeding occur following clots removal. We had successfully removed a large blood clot from our patient via rigid bronchoscopy.

However, rigid bronchoscopy is not always available in all hospital. Therefore other methods should be sought if flexible bronchoscope failed to remove the blood clot. Fogarty arterial embolectomy catheter via the working channel of a flexible bronchoscope had been used with success especially for firmly adherent clot (2). Forgarty catheter can be passed down the working channel of a flexible bronchoscope, along the margin of the obstructed airway and beyond the level of clot. The balloon is then inflated and the catheter withdrawn to dislodge the clot (2). One must remember that any intervention to remove the endobronchial blood clot may induce further bleeding or result in more proximal obstruction. Another advantage of this catheter is to tamponade the affected airway if brisk bleeding occurs. Topical thrombolysis by thrombolytic applied directly on the clot has been used with success (2). The potential complication of this procedure is recurrence of bleeding.

In conclusion, massive haemoptysis can arise from lung abscess and may lead to acute airway obstruction and life-threatening episodes. Rigid bronchoscopy is one of the methods available to successfully evacuate large endobronchial blood clots.

\section{References}

1. de Gregorio MA, Medrano J, Mainar A, et al. Endovascular treatment of massive haemoptysis by bronchial artery embolization: Short-term and long-term follow up over a 15-year period. Arch Bronconeumol 2006; 42: 49-56.

2. Keane LA, Marc AJ, Steven AS. Airway obstruction arising from blood clot. Three reports and a review of the literature. Chest 1999; 115: 293-300.

3. Alexander S, Jeffrey G. Fiberoptic bronchoscopy in the evaluation of lung abscesses. Chest 1985; 87: 489-494.

4. Fredrerick SK, Josef R, Alan FB, et al. Percutaneous Int erventional catheter therapy for lesions of the chest and lungs. Chest 1982; 81: 407-412. 